\title{
Photoabsorption and Carrier Transport Modeling in Thin Multilayer Photovoltaic Cell
}

\author{
František Čajko and Alexander I. Fedoseyev* \\ CFD Research Corporation, 215 Wynn Dr., Huntsville, AL 35805 \\ ph.: (256)726-4928; fax: (256)726-4806 \\ aif@cfdrc.com
}

\begin{abstract}
The paper describes an efficient implementation of a photoabsorption model for modern thin photovoltaic (PV) cells. As the modelling of solar cells constitutes a multiphysic and multiscale problem, a special attention was paid to the speed while retaining a reasonable accuracy. The model is restrained to a normal incidence but accounts for the whole solar spectrum. Applied transfer matrix method yields an accurate distribution of the light intensity in the semiconductor structure. Usage of equivalent parameters makes it possible to simulate both plain semiconductor material and a quantum dot superlattice based material, which is used to enhance the PV device performance.
\end{abstract}

Keywords: photovoltaic cell, photoabsorption, carrier generation, multiple wavelength.

\section{Introduction}

Solar cells answer a call for alternative energy by providing a source of a reliable, long lasting power supply. Most designs of solar cells rely on semiconductor materials based on silicon or III-V semiconductors. Space electronic equipment requires improvements in solar cell efficiency to deliver more efficient, lightweight solar cells. Recently proposed approaches to enhance the efficiency utilize the novel nanomaterials containing quantum dots [1, and new concepts of cell design. A proper models and simulation techniques are needed to speed-up the development on novel solar cell devices and reduce the related expenses.

Modeling of a photoabsorption in solar cells is a complex problem that involves multiphysics simulations of multiscale problem at different levels [2]. Recent trend of using quantum dots to enhance the performance of a solar cell by broadening an absorption spectrum and reducing a recombination rate requires modeling at a quantum level. A spatial distribution of the light intensity in the structure is obtained at a macroscopic level because of the dimensions of the solar cell. Carrier generation and recombination rates are used in transport equations in order to calculate a carrier distribution.

Our goal is to develop a simulation tool NanoTCAD [3] that will accurately predict a performance of a solar cell and allows an inside view that helps in future

^ Corresponding author.

G. Allen et al. (Eds.): ICCS 2009, Part I, LNCS 5544, pp. 755 764, 2009.

(C) Springer-Verlag Berlin Heidelberg 2009 
designs. The NanoTCAD simulator already implements transport equations in semiconductor devices. It can compute a steady-state or a transient. Because of solar cells we are now interested more in the steady-state. A simulation of a typical design takes 100 - 600 thousands volume elements. For a simulation of a small problem without details with only 8,000 elements the computation on $2 \mathrm{GHz}$ Pentium PC takes about 25 seconds and such simulation represents only one point on a I-V curve. Therefore CPU time saving is highly desirable, especially in time dependent problems, e.g. a radiation damage modelling.

This paper is focused on a simplified and yet accurate model of photoabsorption to be included into the simulator. The essential part is to compute an electromagnetic field (EM) distribution inside the solar cell. We want to achieve this without a necessity to implement a full electromagnetic solver that would substantially slow down the $3 \mathrm{D}$ computation.

\section{Geometry of Solar Cells}

We consider a sandwitch structure of a solar cell with upper electrodes organized in a grid and creating rectangular windows for the light to enter into the structure. The incident solar light is a plane wave limited by the window between electrodes impinging the top surface along the normal. A part of the solar cell is depicted in Fig. 1. The aperture defines a horizontal cross section of the beam. It is assumed that the cross section of the beam doesn't vary in the vertical direction due to a shallow geometry of solar cells. Formally, the horizontal area can be split into three parts: (i) the area under the window where a plane wave penetrating in a vertical direction is assumed - carrier transport and photoabsorption occur, (ii) the area outside the window with no light intensity - electron transport but no photoabsorption, (iii) area near the window edge - diffraction and scattering take place. This model is suitable for solar cells because the window is much larger than the wavelength so that diffraction and scattering effects on a border of the cross section can be neglected.

The light propagating through the window of a solar cell can be decomposed into periodic fields

$$
E(x, y, z)=\int \tilde{E}\left(k_{x}, k_{y} ; z\right) e^{i \frac{2 \pi}{\lambda_{0}}\left(k_{x} x+k_{y} y+\beta z\right)} d k_{x} d k_{y}
$$

with value

$$
\beta=\sqrt{\varepsilon_{r}-k_{x}^{2}-k_{y}^{2}}
$$

It is obvious that for $\left(k_{x}^{2}+k_{y}^{2}\right)<<\varepsilon_{r}$, the value of $\beta$ is almost constant, meaning that all waves propagates at about the same phase velocity, and hence, the horizontal profile is changing very slowly. The relative difference of the electric field caused by neglecting $k_{x}, k_{y}$ in the expression above is

$$
\left|\frac{E-E\left(k_{x}=k_{y}=0\right)}{E\left(k_{x}=k_{y}=0\right)}\right|=\left|e^{i \frac{2 \pi}{\lambda_{0}}\left(\beta-\sqrt{\varepsilon_{r}}\right) z}-1\right| \approx\left|\frac{\pi\left(k_{x}^{2}+k_{y}^{2}\right) z}{\lambda_{0} \sqrt{\varepsilon_{r}}}\right|
$$




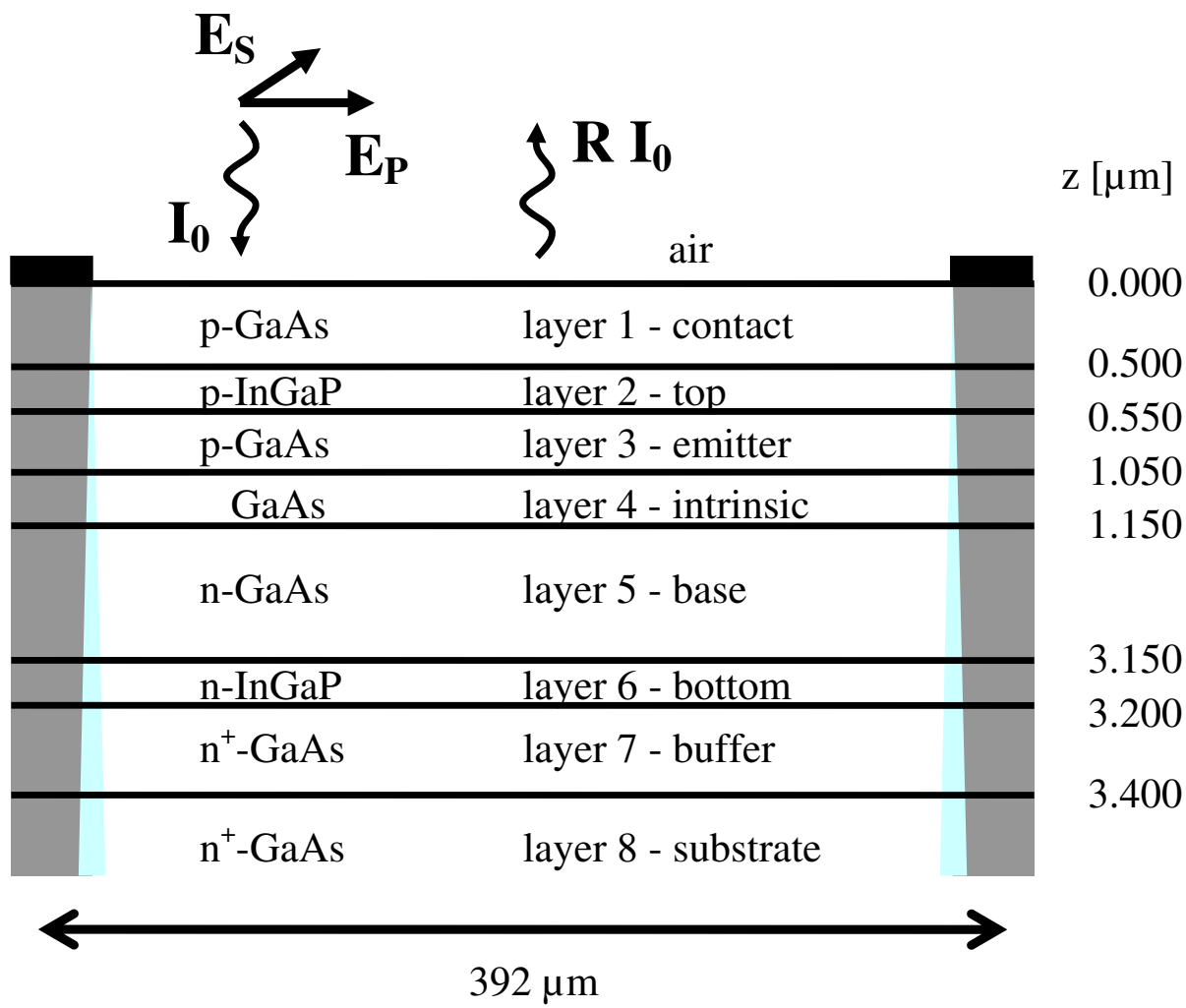

Fig. 1. A schematic multilayer structure with a window for incident light created by electrodes (black). Incident irradiation $I_{0}$, reflected power flux $R I_{0}$ and two polarizations $E_{P}, E_{S}$ of the electric field shown. Shaded areas respresents regions without light.

for components with small $k_{x}$ and $k_{y}$. Considering a typical value of $\sqrt{\varepsilon_{r}}$ to be 3 , it is required that $\left(k_{x}^{2}+k_{y}^{2}\right) z<<\lambda_{0}$. For an aperture with a diameter $a$ the components that matter have wavenumbers up to $2 \pi / a$ (i.e. $\left.\sqrt{\left(k_{x}^{2}+k_{y}^{2}\right)}=\lambda_{0} / a\right)$. For a structure with a height about $\lambda_{0}$ and a window diameter about $1 \mathrm{~cm}$ the estimated relative error in the center of a window that corresponds to one Fourier component is about $10^{-4}$.

\section{Plane Waves in the Structure at a Single Wavelength}

All properties within one layer are assumed to be constant but dependent on a free space wavelength $\lambda_{0}$. Electrooptical properties of each layer are described by a refractive index $n$ and an extinction coefficient $k$. A general solution in the $j^{t h}$ homogeneous layer is a superposition of two counterpropagating waves

$$
\boldsymbol{E}_{j}(z)=\boldsymbol{E}_{0 j}^{+} e^{+i K_{j}\left(z-z_{0 j}\right)}+\boldsymbol{E}_{0 j}^{-} e^{-i K_{j}\left(z-z_{0 j}\right)}
$$


with $e^{-i \omega t}$ time factor. The complex vectors $\boldsymbol{E}_{0 j}^{+}$and $\boldsymbol{E}_{0 j}^{-}$represent the values of plane wave components in a reference depth $z=z_{0 j}$ (chosen at the top of the layer $j$ ) propagating downwards and upwards, respectively. Each propagating wave is a solution of the Helmholtz equation

$$
\nabla^{2} \boldsymbol{E}_{j}(z)+K_{j}^{2} \boldsymbol{E}_{j}(z)=0
$$

with a wavenumber

$$
K_{j}=K_{0}\left(n_{j}+i k_{j}\right)=\frac{2 \pi}{\lambda_{0}}\left(n_{j}+i k_{j}\right)
$$

Given the assumption about geometry and the normal direction of incidence, the EM fields vary only along the normal. Any solution of such problem can be decomposed into two independent polarizations (Fig 1) - $\mathrm{S}\left(E=\hat{y} E_{y}, H=\right.$ $\left.\hat{x} H_{x}\right)$ and $\mathrm{P}\left(E=\hat{x} E_{x}, H=\hat{y} H_{y}\right)$ - that are just a mutual rotation and both are described by the same Helmholtz equation (5).

Natural light is not polarized. It means an equal contribution of both polarizations. As we are interested in the power intensity (proportional to $|\boldsymbol{E}|^{2}=$ $\left|E_{x}\right|^{2}+\left|E_{y}\right|^{2}$ ) and solutions for both polarizations are identical, the formula

$$
E(z)=E_{0}^{+} e^{+i K\left(z-z_{0}\right)}+E_{0}^{-} e^{-i K\left(z-z_{0}\right)}
$$

for a scalar equivalent field intensity $E=|\boldsymbol{E}|$ can be used instead. The overall solution is tailored by applying a continuity boundary conditions across the layer interfaces for tangential components of the electric and magnetic fields assuming one of the polarizations.

The usual approach is to use the transfer matrix method [5]. An electric field at each point is represented by two complex fields $E^{+}$and $E^{-}$. In a homogeneous layer they are given by the subexpressions of (7) or in a matrix form

$$
\left(\begin{array}{c}
E^{+}(z) \\
E^{-}(z)
\end{array}\right)=\left(\begin{array}{cc}
e^{+i K\left(z-z_{0}\right)} & 0 \\
0 & e^{-i K\left(z-z_{0}\right)}
\end{array}\right)\left(\begin{array}{l}
E^{+}\left(z_{0}\right) \\
E^{-}\left(z_{0}\right)
\end{array}\right)
$$

A transition across an interface between two layers is defined by boundary conditions for the electric and magnetic fields

$$
\left(\begin{array}{cc}
1 & 1 \\
1 / Z_{1} & -1 / Z_{1}
\end{array}\right)\left(\begin{array}{c}
E_{1}^{+} \\
E_{1}^{-}
\end{array}\right)=\left(\begin{array}{cc}
1 & 1 \\
1 / Z_{2} & -1 / Z_{2}
\end{array}\right)\left(\begin{array}{l}
E_{2}^{+} \\
E_{2}^{-}
\end{array}\right)
$$

where $Z_{i}$ is an intrinsic impedance of the layer $i$ with refraction index $n_{i}$ and extinction coefficient $k_{i}$ and relates to the intrinsic impedance $Z_{0}$ of vacuum as

$$
Z_{i}=\frac{Z_{0}}{n_{i}+i k_{i}} \approx \frac{377 \Omega}{n_{i}+i k_{i}}
$$

One can find that

$$
\left(\begin{array}{c}
E_{1}^{+} \\
E_{1}^{-}
\end{array}\right)=\frac{1}{2}\left(\begin{array}{c}
1+Z_{1} / Z_{2} 1-Z_{1} / Z_{2} \\
1-Z_{1} / Z_{2} 1+Z_{1} / Z_{2}
\end{array}\right)\left(\begin{array}{c}
E_{2}^{+} \\
E_{2}^{-}
\end{array}\right)
$$


The total electrical field can be reconstructed by assuming no backward propagating wave in the bottom layer (i.e. $E_{0}^{-}=0$ ) and a unit amplitude of the forward wave $\left(E_{0}^{+}=1\right)$. Then, applying matrix operations (8), (11) yield a recursive formulas for layers above

$$
\begin{aligned}
& E_{0, j}^{+}=\left(a E_{0, j+1}^{+}+b E_{0, j+1}^{-}\right) \cdot \exp \left[+i K_{j}\left(z_{0, j}-z_{0, j+1}\right)\right] \\
& E_{0, j}^{-}=\left(b E_{0, j+1}^{+}+a E_{0, j+1}^{-}\right) \cdot \exp \left[-i K_{j}\left(z_{0, j}-z_{0, j+1}\right)\right]
\end{aligned}
$$

with coefficients $a=1+Z_{j} / Z_{j+1}$ and $b=1-Z_{j} / Z_{j+1}$. Once all amplitudes are known, they are scaled in order to match the actual incident irradiance $I_{0}$, i.e. the forward propagating wave in air must be

$$
E_{\text {air }}^{+}=\sqrt{2 Z_{0} I_{0}}
$$

The transfer matrix method calculates the reflectance of the structure for an ideal case. Since the real parameters of semiconductors are not exactly known, and because of a surface roughness and scattering from edges of the window, the experimentally measured reflectance can differ from the calculated one. Fortunately, there is an easy calibration.

Let $I_{u}$ be the incident irradiance and $R_{u}$ a measured reflectance supplied by a user. Then, power $\left(1-R_{u}\right) I_{u}$ transmitted into the structure should be preserved. If the calculated reflectance from the first layer is $R_{0}$, then the equivalent irradiance used in the calculations is

$$
I_{0}=I_{u} \frac{1-R_{u}}{1-R_{0}} .
$$

\section{Carrier Generation Rate}

Linear photoabsorption refers to a phenomenon of capturing a photon that causes a generation of one electron-hole pair (e-h). It can be described by the classical electrodynamics or by the quantum mechanics. In the classical electrodynamics the interaction with material is described by a complex permittivity, or alternatively, by a refraction index and an extinction coefficient. At this level we are able to calculate a light intensity distribution in the material which is important for an energy absorption. The absorbed energy is used for an electron transition and, hence, links both, classical and quantum mechanical, approaches together.

The total carrier generation rate is contributed by all spectral components for which a photoabsorption occurs. First, an expression for a single frequency will be found, then an integration over the spectrum will be discussed. All calculations assume one dimensional layer structure where the field distribution is well known.

\subsection{Single Wavelength}

The horizontal profile of the light intensity is well defined. The absorbed power per unit volume can be calculated directly as

$$
\frac{d P_{\mathrm{ab}}}{d V}=\frac{\omega \varepsilon_{0} \varepsilon^{\prime \prime}}{2}|E|^{2}=2 n k K_{0} \frac{|E|^{2}}{2 Z_{0}}
$$


where $\omega=K_{0} c=2 \pi c / \lambda_{0}$ is an angular frequency of the light, $c$ speed of the light in vacuum, $n+i k$ the complex refraction index, $Z_{0}$ the intrinsic impedance of vacuum and

$$
|E|^{2}=\left|E^{+}(z)+E^{-}(z)\right|^{2}
$$

The electric field $\left(E^{+}+E^{-}\right)$is given by (7). The amplitudes $E_{0}^{+}, E_{0}^{-}$and the reference position $z_{0}$ relate to a layer at the given position $z$. It is easy to show that

$$
|E|^{2}=\left|E_{0}^{+}\right|^{2} e^{-2 k z^{\prime}}+\left|E_{0}^{-}\right|^{2} e^{+2 k z^{\prime}}+2 \Re\left\{E_{0}^{+}\left(E_{0}^{-}\right)^{*} e^{i 2 n z^{\prime}}\right\}
$$

To save space, a shorted the notation

$$
z^{\prime}=K_{0}\left(z-z_{0}\right)
$$

was used. The value of $K_{0}$ is defined by (잠) and the asterisk $\left(^{*}\right)$ denotes a complex conjungated value.

Our simulation tool NanoTCAD uses a volume element method. The following expression is the power $P_{a b}^{c e l l}$ absorbed in one cell of the volume element method for given wavelength $\lambda_{0}$ of the light

$$
P_{\mathrm{ab}}^{\text {cell }}=\int_{\text {cell }} \frac{d P_{\mathrm{ab}}}{d V} d V=A \int_{z_{1}}^{z_{2}} \frac{d P_{\mathrm{ab}}}{d V} d z
$$

The integration over $x$ and $y$ can be taken care of by a simple multiplication by a horizontal cross sectional area $A$ of the cell. The integration along $z$ has to be conducted properly. Knowing positions of the top and bottom walls of the cell, denoted by $z_{1}$ and $z_{2}\left(z_{1}<z_{2}\right)$ and the primitive function of (17) which is

$$
\frac{1}{2 K_{0} k}\left[-\left|E_{0}^{+}\right|^{2} e^{-2 k z^{\prime}}+\left|E_{0}^{-}\right|^{2} e^{+2 k z^{\prime}}\right]+\Im\left\{\frac{E_{0}^{+}\left(E_{0}^{-}\right)^{*}}{K_{0} n} e^{i 2 n z^{\prime}}\right\}
$$

the absorbed power can be expressed as

$$
P_{\mathrm{ab}}^{\text {cell }}=\frac{A n}{2 Z_{0}}\left[\left|E_{0}^{-}\right|^{2} e^{+2 k z^{\prime}}-\left|E_{0}^{+}\right|^{2} e^{-2 k z^{\prime}}+2 \frac{k}{n} \Im\left\{E_{0}^{+}\left(E_{0}^{-}\right)^{*} e^{i 2 n z^{\prime}}\right\}\right]_{z^{\prime}=K_{0}\left(z_{1}-z_{0}\right)}^{K_{0}\left(z_{2}-z_{0}\right)}
$$

The refraction index $n$ for ordinary materials is non-zero, so the expression is well defined. Once the power absorbed in a cell is calculated, the electron-hole generation rate in the cell can be obtained as [4]

$$
d N_{\mathrm{e}-\mathrm{h}} / d t=\frac{\eta}{\hbar \omega} P_{\mathrm{ab}}^{\mathrm{cell}}
$$

where the internal quantum efficiency $\eta$ is a property of the material and $\hbar \omega$ is energy of photons at a given wavelength. The equation represents the total generation rate of the whole volume element at the given wavelength. 


\subsection{Spectrum of the Solar Light}

The solar radiation outside the Earth's atmosphere is described by air mass 0 (AM0) spectrum. The total amount of delivered power is known as a solar constant $S$. Its value was determined to be $1366.1 \mathrm{~W} / \mathrm{m}^{2}$ 6]. We use 2000 ASTM Standard Extraterrestrial Spectrum Reference (E-490-00) obtained from [6 for simulation of solar cells. Abrupt (noise like) changes in the spectrum (Fig 2) can prevent an observation of a convergence of a numerically evaluated spectral integral because of a random choice of sample points. Therefore a cumulative spectral density $\Phi$ was introduced. An irradiance in interval of energies $E_{1} \ldots E_{2}$ is then

$$
d \Phi\left(E_{1}, E_{2}\right)=\left|\Phi\left(E_{1}\right)-\Phi\left(E_{2}\right)\right|
$$

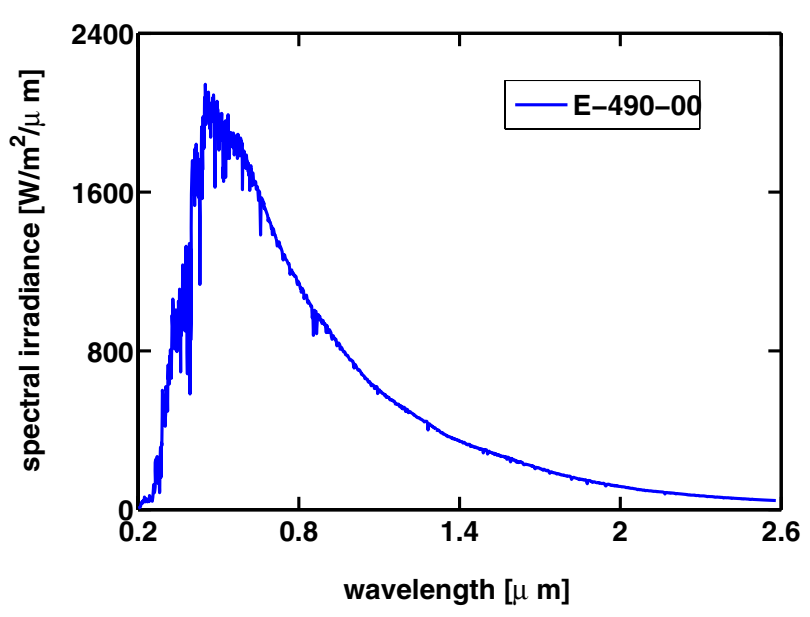

Fig. 2. Part of the AM0 spectrum. Data from E_490_00.

\subsection{Multiple Wavelengths}

Since the system is linear, the field distribution (7) can be calculated under an assumption of a unit incident irradiance $I_{0}$ yielding the absorbed power within one cell at the specified frequency calculated from (22) to be $P_{1}^{c e l l}$. Then, it is multiplied by a true incident power within a small interval of energies $d E$

$$
d P_{\mathrm{ab}}^{\mathrm{cell}}=P_{1}^{\mathrm{cell}} \frac{d \Phi(E)}{d E} d E=P_{1}^{\mathrm{cell}} d \Phi(E)
$$

For a multicolor light, the e-h pair generation rate is an integral of the expression (23) over the whole spectrum of the incident light (e.g. AM0)

$$
d N_{\mathrm{e}-\mathrm{h}}^{\text {cell }} / d t=\int \eta(E) \frac{P_{1}^{\text {cell }}(E)}{E} \frac{d \Phi(E)}{d E} d E
$$


Notice that also $P_{1}^{\text {cell }}$ has to be recalculated for every $\lambda$ according to (22). There are two reasons for that: (i) material properties are frequency dependent and (ii) optical distance (phase difference) between layers changes as well.

The integral (26) will be evaluated numerically. The lower limit is given by the lowest energy with a nonzero quantum efficiency $\eta$. The upper limit is given by the spectrum itself. We choose to cut-off the spectrum at $99 \%$ of the total energy.

The integration of (26) can be performed in a semianalytical way: (i) the spectrum is split into $M$ subintervals, (ii) an analytical integration is performed in each subinterval, (iii) partial results are summed together

$$
d N_{\mathrm{e}-\mathrm{h}}^{\mathrm{cell}} / d t=\int_{\hbar \omega_{\mathrm{START}}}^{\hbar \omega_{\mathrm{STOP}}} G_{1}^{\mathrm{cell}} d \Phi=\sum_{i=0}^{M-1} \int_{\hbar \omega_{i}}^{\hbar \omega_{i+1}} G_{1}^{\mathrm{cell}} \frac{d \Phi}{d E} d E
$$

Here, $G_{1}^{\text {cell }}=\eta(E) P_{1}^{\text {cell }}(E) / E$ is a generation rate in a cell for a monochromatic light with a unit incident irradiance, $\hbar \omega_{i}$ are the split points and $E$ is an integration variable that runs over all photon energies $\hbar \omega$.

In order to do the analytical integration on subintervals the following approximations were made

1. the spectral density $d \Phi / d E$ is constant on each subinterval

2 . the generation rate can be approximated by a piecewise linear function

$$
G_{1}^{\mathrm{cell}}(\hbar \omega) \approx G_{1}^{\mathrm{cell}}\left(\hbar \omega_{i}\right)+\frac{G_{1}^{\mathrm{cell}}\left(\hbar \omega_{i+1}\right)-G_{1}^{\mathrm{cell}}\left(\hbar \omega_{i}\right)}{\hbar \omega_{i+1}-\hbar \omega_{i}}\left(\hbar \omega-\hbar \omega_{i}\right)
$$

This approach results in a simple expression

$$
d N_{e-h}^{\mathrm{cell}} / d t=\sum_{i=0}^{M-1} \frac{G_{1}^{\mathrm{cell}}\left(\hbar \omega_{i}\right)+G_{1}^{\mathrm{cell}}\left(\hbar \omega_{i+1}\right)}{2} d \Phi\left(\hbar \omega_{i}, \hbar \omega_{i+1}\right)
$$

\section{Numerical Results and Discussion}

The modeling of 3D electromagnetic field calls for accurate models of semiconductor properties. The refraction and extinction coefficients necessary to calculate an absorption rate are usually obtained by the ellipsometry technique and can be found in the literature $7[8$.

The model was implemented into Matlab ${ }^{\mathrm{TM}}$ for testing purposes. $99 \%$ of the AM0 spectrum was considered in the computation. The spectrum was further cut-off at the longest wavelength at which a photoabsorption occurs yielding a range of photon energies $1.375 \mathrm{eV}-4.16 \mathrm{eV}$. This range was divided into $M$ subinterval. An convergence of the total deposited charge was observed and $M=100$ chosen based on $1 \%$ error. A distribution of a e-h generation rate $G$ was computed on a standard $3 \mathrm{GHz}$ PC within a fraction of second. Unlike in the NanoTCAD implementation, the generation rate was calculated along a 


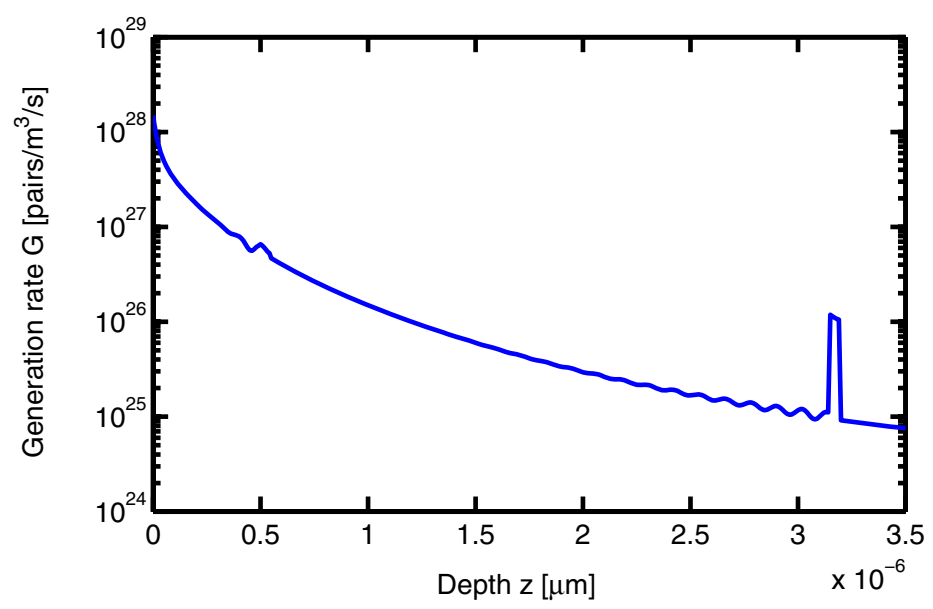

Fig. 3. A carrier generation rate in the solar cell structure along a vertical axis in the center of the window

vertical axis only in the center of the window. The actual implementation has to compute $G$ for every cell of the volume element method.

It is not possible to calculate the generated photocurrent without solving transport equations. Therefore only its order was calculated assuming $60 \%$ external efficiency [4] and compared with a typical value of $10 \mathrm{~mA} / \mathrm{cm}^{2}$ for $\mathrm{GaAs}$ solar cell.

The testing model comprises of a structure in Fig. 1. The cumulative generation rate per unit cross section saturates at $1.46 \times 10^{21}$ pairs $/ \mathrm{m}^{2} / \mathrm{s}$ what represent $100 \%$ external quantum efficiency. Since most of the structure is created from GaAs a factor of 0.6 was used to account for the external quantum efficiency, thus yielding $0.85 \times 10^{21}$ pairs $/ \mathrm{m}^{2} / \mathrm{s}$ or $13.6 \mathrm{~mA} / \mathrm{cm}^{2}$ which is in the expected order.

For illustration purposes a distribution of a volume generation rate $G$ is displayed in Fig. 3. In each material the generation rate decays exponentially with a corresponding attenuation constant. The wiggles observed between 2 and $3.15 \mu \mathrm{m}$ are due to an interference of forward and backwards waves. It seems that the main source of the reflection is the bottom InGaP layer that manifest itself by a $50 \mathrm{~nm}$ wide spike of increased light absorption. Similar behavior of a smaller relative amplitude is observed above the top InGaP layer.

\section{Conclusions}

A photoabsorption model for solar cells and technical details of the computation were presented. Extra assumptions were made for solar cell applications: the structure irradiated by a plane wave, a size of the solar cell window much larger than the wavelength, scattering on the edges of the window and on 
inhomogeneities as well as a scattering due to a photon recycling neglected. The material properties were described by an index of refraction and an extinction coefficient. In this paper are considered to be known.

The described approach resulted in a Matlab ${ }^{\mathrm{TM}}$ implementation of the photoabsorption model for testing purposes and yielded preliminary results. An absorption rate for one test case is presented in the previous section. The e$\mathrm{h}$ generation is mostly defined by the material properties. The total deposited charge of $13.6 \mathrm{~mA} / \mathrm{cm}^{2}$ is found to be in the expected order of magnitude.

The full implementation into NanoTCAD is still an ongoing work. The test cases include plain binary and ternary III-V semiconductors but the model is intended for quantum dots superlatices (QDS) as well. Equivalent parameters of QDS have to be calculated separately.

Acknowledgments. This work has been supported by AFOSR under Contract No. FA9550-07-C-0059 and by NASA under Contract No. NNC07CA20C.

\section{References}

1. Shao, Q., Balandin, A.A., Fedoseyev, A.I., Turowski, M.: Intermediate-band solar cells based on quantum dot supracrystals. App. Phys. Let. 91(16) (2007)

2. Fedoseyev, A.I., Turowski, M., Wartak, M.S.: Kinetic and Quantum Models in Simulation of Modern Nanoscale Devices. In: Balandin, A.A., Wang, K.L. (eds.) Handbook of Semiconductor Nanostructures and Devices. American Scientific Publishers, Los Angeles (2006)

3. CFDRC, NanoTCAD web site (2008), http://www.cfdrc.com/bizareas/microelec/micro_nano/

4. Sze, S.M.: Physics of Semiconductor Devices, 2nd edn. John Wiley \& son, New York (1981)

5. Piprek, J.: Semiconductor Optoelectronic Devices: Introduction to Physics and Simulation. Academic Press, New York (2003)

6. 2000 ASTM Standard Extraterrestrial Spectrum Reference E-490-00, Renewable Resource Data Center, National Renewable Energy Laboratory, http://rredc.nrel.gov/solar/spectra/am0/ASTM2000.html

7. Palik, E.D. (ed.): Handbook of Optical Constants of Solids. Academic Press, New York (1985)

8. Adachi, S.: Optical dispersion relations for GaP, GaAs, GaSb, InP, InAs, InSb, $\mathrm{Al}_{x} \mathrm{Ga}_{1-x} \mathrm{As}$, and $\mathrm{In}_{1-x} \mathrm{Ga}_{x} \mathrm{As}_{y} \mathrm{P}_{1-y}$. J. Appl. Phys. 66, 6030-6040 (1989) 